\title{
The road less traveled
}

\section{Traditional approaches for salugaging peri- Traditional odonatly irovered teeth have} faced the test of whether or not the treatment modalities can guarantee tooth survival for more than a couple of decades. This challenge stems from the most recent encouraging reports of a success rate of over 15 years of implant therapy to treat partial edentulism.

The main criticism of traditional periodontal therapy targets the predictive value of regenerative treatment modalities, especially in restoring 1-wall intrabony defects with an insufficient amount of bone fill that falls far short of the size of the osseous defect to be eradicated. The only exception could be demonstrated in cases of true 3-wall intrabony defects. However, we have not been able to manage intrabony lesions to the extent of reconstituting of the physiological contour of the osseous scallop. In addition, clinicians have not yet fully developed a strategy for resecting molars that allows them to survive more than 10 years without root fracture or recurrent periodontal disease.

We have not yet fully introduced the concept of occlusion in the management of seriously compromised cases of advanced periodontal disease. Furthermore, we have not defined a protocol with a scientific basis for a so-called "periodontal prosthesis" as an alternative to implant-supported overdenture or removable partial denture. 
The road less traveled extends ahead of us and invites us to explore it. Pursuing a higher level of predictability and longevity demands our commitment. Otherwise, we may succumb to being generalists, losing our professional identity as periodontal specialists. Let us take the road less traveled.

\section{Jeomil Choi}

Department of Periodontology, Pusan National University School of Dentistry, Beomeo-ri,

Mulgeum-eup, Yangsan 626-870, Korea

E-mail: jrapa@pusan.ac.kr, Tel: +82-55-360-5200, Fax: +82-55-360-5194 